\title{
Calibration of Voltage Transformers and High-Voltage Capacitors at NIST
}

\section{William E. Anderson \\ National Institute of Standards and Technology, Gaithersburg, MD 20899}

\begin{abstract}
The National Institute of Standards and Technology (NIST) calibration service for voltage transformers and highvoltage capacitors is described. The service for voltage transformers provides measurements of ratio correction factors and phase angles at primary voltages up to $170 \mathrm{kV}$ and secondary voltages as low as $10 \mathrm{~V}$ at $60 \mathrm{~Hz}$. Calibrations at frequencies from $50-400 \mathrm{~Hz}$ are available over a more limited voltage range. The service for high-voltage capacitors provides measurements of capacitance and dissipation factor at applied voltages ranging from
\end{abstract}

$100 \mathrm{~V}$ to $170 \mathrm{kV}$ at $60 \mathrm{~Hz}$ depending on the nominal capacitance. Calibrations over a reduced voltage range at other frequencies are also available. As in the case with voltage transformers, these voltage constraints are determined by the facilities at NIST.

Key words: calibration; capacitors; dissipation factor; electric power; electrical standards; NIST services; voltage transformers.

Accepted: February 15, 1989

\section{Introduction}

This paper describes the National Institute of Standards and Technology (NIST) methodology for calibrating high-voltage capacitors and transformers. This should benefit NIST clients in several ways. First, by understanding how NIST makes these measurements, the clients might be able to define weaknesses in their own measurement procedures and correct them. Second, the clients should be able to make better use of the data in the calibration report (e.g., to understand what is meant by the uncertainty statement). Third, the clients should be able to better specify the required test conditions so that information more pertinent to their needs can be obtained at a lower cost.

This paper describes two different calibration services: high-voltage capacitors and voltage transformers. At NIST these two services are performed using the same equipment. In fact, in order to calibrate a voltage transformer, one of the steps is to measure the ratio of two capacitors. The two services are therefore discussed in parallel.

There are several different ways to measure the ratio and phase angle of a voltage transformer. Harris [1] categorizes them as the direct versus comparative methods and within these two classifications either the deflection or null measurement technique. A direct measurement is defined here as a measurement in which the quantity of interest can be determined without a comparison to some absolute standard.

In the "direct deflection method" the primary and secondary voltage vectors are each directly measured. This approach is, in general, of most value for lower voltage transformers (i.e., primary voltages of order $100 \mathrm{~V}$ ). Even then more accurate, less difficult measurements can be made using one of the other techniques. 
In the past NIST had used a "comparative null method" to calibrate voltage transformers. The unknown transformer was compared to a NIST reference transformer using a voltage comparator consisting of a variable resistive divider and a mutual inductor. Reference transformers were available with ratios ranging from $1 / 1$ up to $2000 / 1$. Measurement uncertainties in the comparison of the unknown transformer with the reference transformer were $\pm 0.01 \%$ for ratio and \pm 0.3 minutes for phase angle. The ratio and phase angle of the reference transformers were known to about the same accuracy. There are several disadvantages to this approach. Since the comparator has a limited range, several reference transformers must be available to cover the anticipated users' needs. The ratio and phase angles of each one of these transformers must be carefully determined over the secondary voltage range of interest. These transformers then have to be rechecked at regular intervals to determine if the ratios and phase angles have changed.

If a direct measurement method were available that was sufficiently accurate and straightforward to make the calibration of these reference transformers a simple task, then that method could be used to measure the client's transformer directly. At NIST, the "direct null method" in use originally involved balancing the secondary of the reference transformer against the output of a resistive divider used in conjunction with a variable mutual inductor to provide phase angle balance. Such a measurement was difficult because the resistive divider ratio changed with heating. Since the late 1960 s a "direct null method" has been available that is straightforward and accurate and is now used at NIST in place of comparative methods using reference transformers.

Capacitors are invariably measured by balancing the unknown capacitor against a known standard using some type of bridge arrangement. There are a variety of such bridges described in the literature [2]. The one most used in high-voltage applications in the last 60 years is the Schering bridge (fig. 1). The two high-voltage arms of this bridge consist of the standard and unknown capacitors. The two low voltage arms are resistors (one has a parallel capacitor for phase angle balance).

The main limitation of the Schering bridge is that the low side of the unknown and standard capacitors are not at ground potential at bridge balance. Therefore, without carefully guarding the bridge components, stray currents can affect the bridge accuracy. The voltage applied to the shields to eliminate these stray currents must be adjusted for both magnitude and phase. Unfortunately this procedure is not perfect and bridge accuracy is consequently affected. Another limitation of the Schering bridge is the inherent inaccuracy of the resistance ratio of the two low-voltage arms.

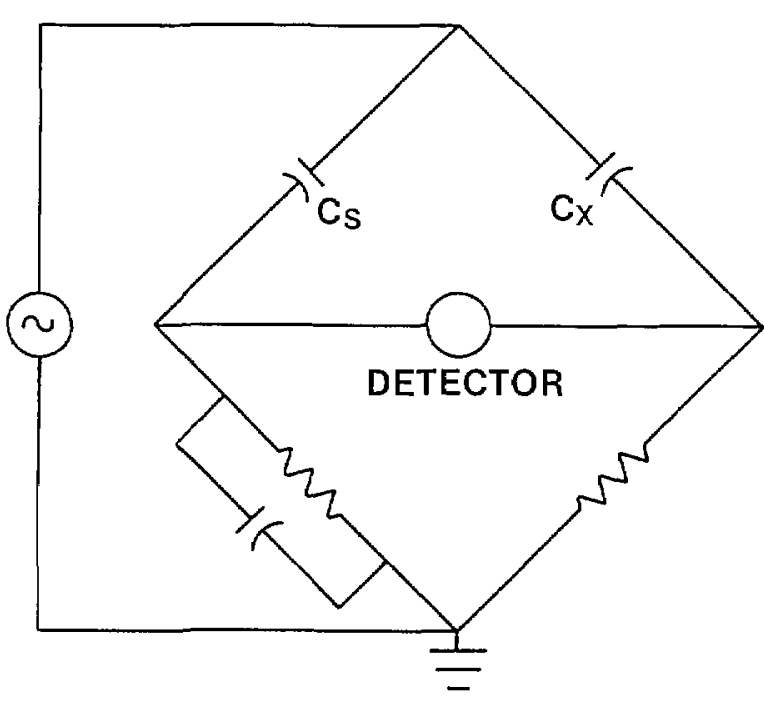

Figure 1. Schering bridge.

The current comparator bridge developed by Kusters and Petersons [3] allows the intercomparison of two capacitors with their low-voltage terminals at ground potential, thereby eliminating the main objection in using the Schering bridge. This bridge, used in both voltage transformer and capacitor calibrations, will be described in some detail in section 4. There is an important distinction between the calibration of voltage transformers and capacitors at NIST. The voltage transformer calibration is of the direct null type, and the capacitor calibration is of the comparative null type. In other words, the accuracy of the capacitance measurements ultimately depends on the uncertainty in assigning a value to a standard capacitor. The standard capacitor used in this service is directly traceable to the calculable cross capacitor [4] which, in turn, is known in terms of the fundamental unit of length.

The remainder of this paper is divided into the following subject areas: voltage transformers and capacitors covered by the service, measurement methodology, measurement instrumentation, and analysis of uncertainties. The contents of this paper plus the cited references should provide the reader with a fairly complete description of the voltage transformer and high-voltage capacitor calibration services at NIST. 


\section{Range of Services}

The NIST measurement capabilities are summarized in table 1 and discussed in more detail below.

Table 1. Measurement capability

\begin{tabular}{ccc}
\hline \hline Voltage transformers- $-60 \mathrm{~Hz}$ & \\
Primary voltage & Secondary voltage & Phase angle \\
$50-170,000 \mathrm{~V} \mathrm{rms}$ & $>50 \mathrm{~V} \mathrm{rms}$ & $<11 \mathrm{mrad}$ \\
Capacitors $-60 \mathrm{~Hz}$ & & \\
Applied voltage & Capacitance & Dissipation factor \\
$50-170,000 \mathrm{~V} \mathrm{rms}$ & $10 \mathrm{pF}-0.001 \mathrm{~F}$ & $<0.011$ \\
\hline
\end{tabular}

Total power must be less than $50 \mathrm{kVA}$.

\subsection{Voltage Transformers}

Presently, voltage transformers (assuming they are of sufficient quality to be used as laboratory standards) with primary voltages up to $170 \mathrm{kV}$ at a frequency of $60 \mathrm{~Hz}$ can be calibrated at NIST. This maximum voltage is imposed by the supply transformer and not by limitations in the measurement instrumentation. Therefore, this constraint should not be considered rigid and clients should contact the NIST about present physical limitations.

The largest portion of the voltage transformers submitted to NIST are calibrated with total estimated uncertainties of \pm 300 parts per million $(\mathrm{ppm})$ in ratio, and $\pm 0.3 \mathrm{mrad}$ in phase angle. These transformers are of sufficient quality to be considered transfer standards. Historically these transformers have shown excellent long-term stability, rarely changing by more than $100 \mathrm{ppm}$ in ratio or $0.1 \mathrm{mrad}$ in phase (at or below rated burden) for periods as long as 30 years or more. In general, the voltage and burden dependence of these transformers are the major contributors to the measurement uncertainties. These uncertainties ( $\pm 300 \mathrm{ppm}$ for ratio, $\pm 0.3 \mathrm{mrad}$ for phase angle) meet the accuracy requirements of most NIST clients.

Voltage transformers of a higher accuracy class often serve as transfer standards for manufacturers of voltage transformers and voltage transformer test sets (voltage comparators). The estimated uncertainties for these transformers are $\pm 100 \mathrm{ppm}$ in ratio, and $\pm 0.1 \mathrm{mrad}$ in phase angle. They are generally designed for use with very small burdens ( $<15$ volt-amperes).

The above discussion for voltage transformers assumes a voltage at a frequency of $60 \mathrm{~Hz}$. The National Institute of Standards and Technology has some capability to calibrate voltage transformers from about $50 \mathrm{~Hz}$ to $400 \mathrm{~Hz}$ (at the lower voltage and power ranges). Such calibrations are infrequent and clients interested in these voltage ranges and measurement uncertainties should contact NIST directly.

\subsection{Capacitors}

The maximum voltage for capacitor calibrations is presently $170 \mathrm{kV}$ at $60 \mathrm{~Hz}$. The restrictions are imposed by the supply transformer and not by limitations in the measurement instrumentation. Therefore, this constraint should not be considered time invariant and clients should contact NIST about present physical limitations.

The maximum power available is $50 \mathrm{kVA}$ (i.e., $C<50,000 /\left\{2 \pi 60 V^{2}\right\}$ where $V$ is the applied voltage and $C$ is the capacitance). In order to energize the capacitors a resonant circuit is often required to couple the necessary energy into the client's capacitor. Since this requires the availability of an assortment of series and parallel inductors and capacitors, there are undoubtedly some capacitors that, despite having a burden of less than $50 \mathrm{kVA}$, cannot be calibrated. The client should contact NIST before submitting a capacitor for calibration. As with voltage transformers, NIST restricts its calibration services to those devices of sufficient quality to be used as transfer standards. This in general depends upon the stability of the capacitor (i.e., whether the measured capacitance and dissipation factor are intrinsic properties of the device itself or instead are largely a function of conditions at the time of the calibration). For example, small two-terminal capacitors (less than $10,000 \mathrm{pF}$ ) may be significantly influenced by stray capacitance in the measurement circuit. There are cases, however, where one component (capacitance or dissipation factor) is stable and the other is not. For example, power factor capacitors often have relatively stable dissipation factors but have capacitances that vary significantly with applied voltage (even demonstrating hysteresis effects) and temperature. In this case a calibration of dissipation factor would be meaningful. It also is important that the capacitors have connectors ${ }^{1}$ that are generally available, e.g., BNC, GR, UHF, BPO, or Type $\mathbf{N}$.

\footnotetext{
${ }^{1}$ Certain commercial products are identified to adequately specify the experimental procedure. In no case does such identification imply recommendation by NIST, nor does it imply the products are the best available.
} 
The most accurate capacitor calibrations have an uncertainty of \pm 25 ppm for capacitance and an uncertainty of $\pm 5 \times 10^{-6}$ for dissipation factor. For capacitors with large dissipation factors, the dissipation factor uncertainty is generally at least $\pm 1 \%$ of the measured value $\pm 5 \times 10^{-6}$. The uncertainty in the capacitance value and the dissipation factor can be largely a function of the stability of the capacitor.

\section{Measurement Methodology \\ 3.1 Basic Measurement Circuits}

The current comparator bridge used to calibrate voltage transformers and high-voltage capacitors will be discussed in considerable detail in section 4 . A brief discussion of this bridge will be presented here in order to facilitate understanding of the NIST measurement methodology. A simplified circuit for measuring the ratio of two capacitors is shown in figure 2 . (The active circuitry to achieve dissipation factor balance is not included.) At balance

$$
\frac{V 2 \pi f C_{\mathrm{x}} N_{\mathrm{x}}}{N_{\mathrm{D}}}=\frac{V 2 \pi f C_{\mathrm{s}} N_{\mathrm{s}}}{N_{\mathrm{D}}}
$$

where $f$ is the frequency. This can be rewritten

$$
C_{\mathrm{x}}=\frac{N_{\mathrm{s}}}{N_{\mathrm{x}}} C_{\mathrm{s}}
$$

The simplified circuit for measuring the ratio of voltage transformers is shown in figure 3. At balance

$$
\frac{V_{\mathrm{p}} 2 \pi f C_{\mathrm{p}} N_{\mathrm{x}}}{N_{\mathrm{d}}}=\frac{V_{\mathrm{s}} 2 \pi f C_{\mathrm{s}} N_{\mathrm{s}}}{N_{\mathrm{d}}}
$$

or,

$$
\frac{V_{\mathrm{p}}}{V_{\mathrm{s}}}=\frac{N_{\mathrm{s}} C_{\mathrm{s}}}{N_{\mathrm{x}} C_{\mathrm{p}}}
$$

The ratio of the two capacitors in eq (2) can be measured using the circuit of figure 2 .

The measurement of a voltage transformer or a capacitor both involve the measurement of the ratio of two standard capacitors. The measurement of capacitors will be discussed below followed by a discussion on the measurement of voltage transformers.

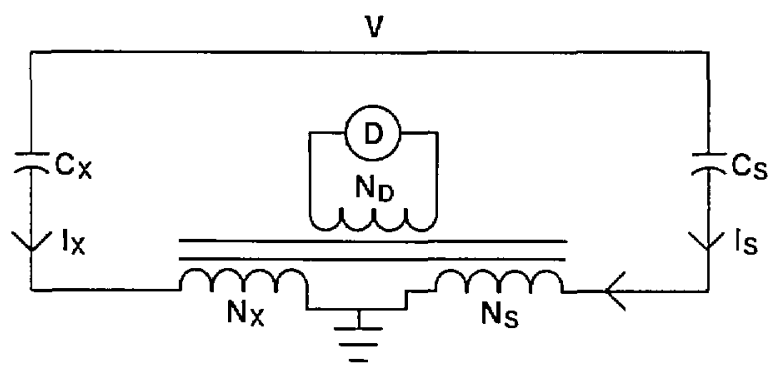

Figure 2. Basic measurement circuit for the calibration of a high-voltage capacitor.

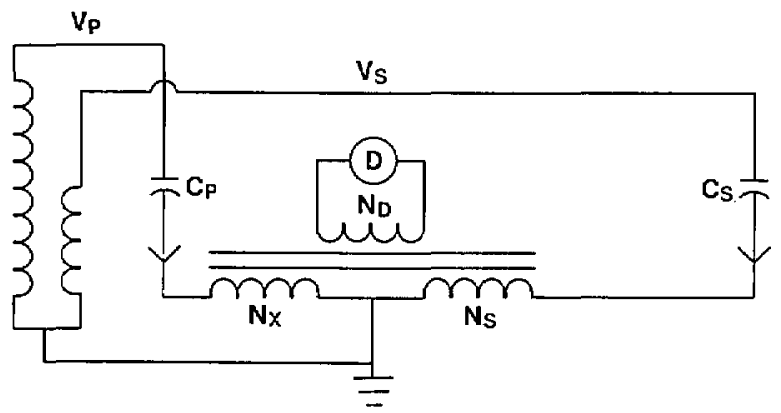

Figure 3. Basic measurement circuit for the calibration of a voltage transformer.

\subsection{Capacitors}

3.2.1 General Measurement Technique Capacitors are measured by balancing the current through the capacitor under test against the current through a standard air or compressed gas capacitor as shown in figure 2. Large capacitors $(>1 \mu \mathrm{F})$ necessitate a four-terminal measurement as shown in figure 4 . This measurement will be discussed in section 4 . The four-terminal measurement eliminates the effect of leads in the measurement of capacitance and dissipation factor.

3.2.2 Information Necessary to Initiate Calibration The client usually only needs to specify the voltage and the frequency. For small capacitors $(10,000 \mathrm{pF}$ or less), it is essential that the lowvoltage electrode and the conductor leading to the measurement instrumentation be shielded by a grounded conductor. Otherwise, the stray capacitance may cause significant measurement error. The National Institute of Standards and Technology requires some sort of standard connector (BNC, UHF, GR, BPO, or Type $\mathbf{N}$ ) at the lowvoltage terminal in order to connect to the measurement system. Larger capacitors do not need to 
be shielded but must be measured as a four terminal admittance because of the non-negligible lead impedance. A description of how this measurement is done will be covered in section 4 . Capacitors must be stable and reproducible in order to be considered standards and hence warrant a NIST calibration. Power factor capacitors (large capacitors used to tune distribution lines, etc.) are often special cases. Their dissipation factors (in-phase component of the current divided by quadrature component) are often quite stable but their capacitance values are often not. Because of the importance of these capacitors to the electrical industry, they are often acceptable for calibration even though they do not meet normal stability requirements.

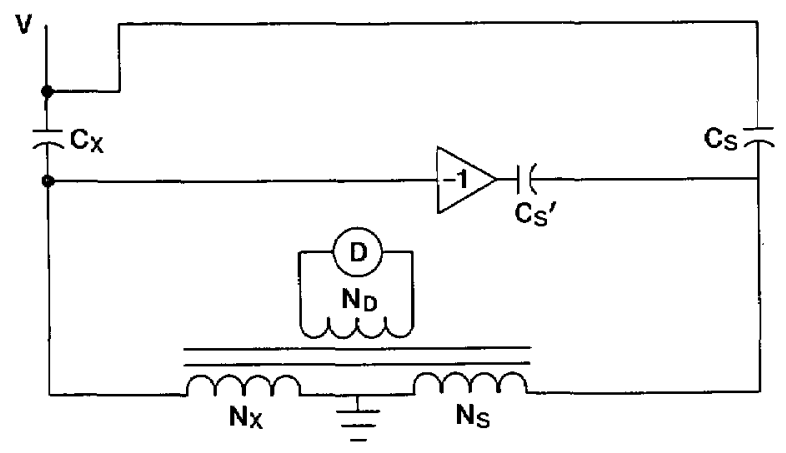

Figure 4. Basic measurement circuit for the four-terminal calibration of large capacitors.

Although the instrumentation has been used to calibrate a million-volt standard capacitor at rated voltage, the instrumentation does impose some limitations on the voltage applied to the capacitor. The only limitation on the maximum voltage is that the current through the standard capacitor should be no larger than $10 \mathrm{~mA}$. In order to have reasonable sensitivity, the current should be at least $10 \mu \mathrm{A}$. The current through the client's capacitor can range from $10 \mu \mathrm{A}$ to $1000 \mathrm{~A}$.

3.2.3 Voltage Dependence For the calibration of both capacitors and voltage transformers, the voltage coefficient of the standard capacitor is important. The unit of capacitance at NIST is maintained at low voltage. This value must be transferred to the high-voltage standard capacitors at their working voltages. At NIST, considerable work was done to modify a commercial highvoltage standard capacitor to minimize its voltage coefficient and to determine the magnitude of that voltage coefficient [5]. The National Institute of
Standards and Technlogy was able to demonstrate that, if care is taken, a well-designed standard capacitor should change capacitance by only a few ppm from 0 to $300 \mathrm{kV}$. A more recent paper also discusses the problem of the voltage dependence of standard capacitors and describes an international comparison of high-voltage capacitor measurements [6]. (This paper also discusses the effect of shipping and handling on the measured capacitance of a commercial standard capacitor.) The voltage dependence of a compressed gas capacitor principally arises from the coulombic attraction of the two electrodes and is hence quadratic in nature. The capacitor should be expected to vary only slightly at lower voltages. Therefore, a capacitor rated at $200 \mathrm{kV}$ should be quite effective in measuring the voltage dependence of another capacitor rated at $20 \mathrm{kV}$.

3.2.4 Temperature Dependence Another concern is the temperature dependence of the high-voltage standard capacitor. The typical dependence is about $+20 \mathrm{ppm} /{ }^{\circ} \mathrm{C}$. This dependence arises solely from the thermal expansion of the components of the capacitor. Since $C$ is directly proportional to the electrode area and inversely proportional to the electrode separation, the thermal coefficient of the standard capacitor is proportional to the linear coefficient of expansion. Although the laboratories at NIST are fairly stable in temperature, the comparison of the high-voltage standard capacitor to the low-voltage standard (which has a thermal coefficient of $2 \mathrm{ppm} /{ }^{\circ} \mathrm{C}$ ) is done at the beginning and conclusion of the measurement process. The average value is then used in order to minimize the problem associated with this thermal drift.

3.2.5 Gas-Density Dependence Compressed gas standard capacitors can have an additional source of error associated with gas leakage. Values of $\partial C / \partial P$ (to first order in pressure) measured at a temperature of $22.8^{\circ} \mathrm{C}$ are shown in table 2 for three different gases [6].

Table 2. Gas density dependence

\begin{tabular}{lc}
\hline \hline Gas & $\begin{array}{c}\partial C / \partial P \text { at } T=22.8^{\circ} \mathrm{C} \\
\text { (units of picofarads/pascal) }\end{array}$ \\
\hline $\mathrm{SF}_{6}$ & $(2.012 \pm 0.022) \times 10^{-6}+\left[(5.1 \pm 0.6) \times 10^{-13}\right] P$ \\
$\mathrm{CO}_{2}$ & $(0.903 \pm 0.015) \times 10^{-6}+\left[(1.4 \pm 0.4) \times 10^{-13}\right] P$ \\
$\mathrm{He}$ & $(0.075 \pm 0.004) \times 10^{-6}+\left[(0.2 \pm 0.1) \times 10^{-13}\right] P$ \\
\hline
\end{tabular}

The gas pressure, $P$, is in units of pascals and the capacitance in picofarads. For a $100-\mathrm{pF}$ capacitor with $\mathrm{SF}_{6}$ as the dielectric gas, a 1-psi (6900-Pa) 
leak would cause the capacitance to decrease by about $140 \mathrm{ppm}$. It must be stressed that this change is valid only if the pressure change is caused by the loss of gas and not by the lowering of the gas temperature. As can be seen in table 2, the gas density coefficient is largest for $\mathrm{SF}_{6}$. Clients using compressed gas capacitors for standards might be advised to monitor the gas pressure with a good quality pressure gauge. Leaking $\mathrm{SF}_{6}$-filled capacitors should be checked often against a good lowvoltage standard.

\subsection{Voltage Transformers}

3.3.1 Information Necessary to Initiate Calibration In order to calibrate a voltage transformer, several different parameters must be specified: frequency; windings and/or range; secondary voltage; and burden or impedance across the secondary winding. In some cases, for example when there is a tertiary winding, additional parameters may be required.

3.3.2 Labeling of Terminals There are some standard conventions as to which of the primary and secondary taps are to be at low or ground potential and which are to be at rated voltage. Some transformers have one tap of the secondary and one tap of the primary winding marked by a " \pm ". These two taps are connected together and to ground potential. Some transformers use the designators $\mathrm{H} 1$, $\mathrm{H} 2$ for the primary taps, and X1, X2 (and $\mathrm{Y} 1$ and $Y 2$ for the transformers with two secondaries) for the secondary taps. Sometimes the secondary winding has a third tap, X3. By convention the primary and secondary taps with the largest number are connected together and to ground. If the client wants some other arrangement, NIST should be notified prior to the calibration.

3.3.3 Load Imposed by NIST Measurement System The basic measurement circuit is shown in figure 5. The two capacitors shown are threeterminal standard capacitors. Their dissipation factors are typically less than $5 \times 10^{-6}$. The capacitor connected to the secondary usually has the nominal value of $1000 \mathrm{pF}$. Therefore, for $60-\mathrm{Hz}$ measurements, the capacitor imposes a negligible load $(2.7 \mathrm{M} \Omega$ or 0.005 volt-amperes at $120 \mathrm{~V})$ on the voltage transformer. Negligible in this case means that the effect of this burden on the measured ratio and phase angle can not be observed at the ppm level. The digital voltmeter (DVM) in figure 5 has an estimated uncertainty of less than $\pm 0.5 \%$ of the reading and measures true-rms ac volts. The internal impedance of the DVM is equal to or greater than one megohm.

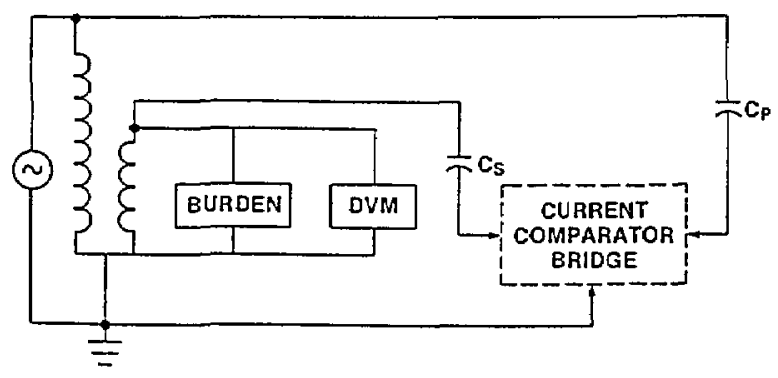

Figure 5. Basic measurement circuit for the calibration of a voltage transformer with a digital voltmeter (DVM) and secondary burden.

3.3.4 Possible Errors Caused by Improper Wiring The wiring of the circuit shown in figure 5 is critical. For example, it is important that the two capacitors be connected directly to the primary and secondary terminals of the transformer. Consider instead figure 6. The capacitor $C_{\mathrm{s}}$ is connected to the burden and the DVM instead of directly to the secondary terminal of the transformer. If the secondary burden were an ANSI standard burden $\mathrm{ZZ}$ ( $36 \Omega$ at $120 \mathrm{~V}$, see table 3 ) and the resistance of the lead connecting the burden to the transformer were $10 \mathrm{~m} \Omega$, the incorrect wiring shown in figure 6 would cause a error in the transformer ratio measurement of about $0.03 \%$. For higher impedance burdens this becomes less of a problem but, in general, one must take precautions to avoid including the voltage drop in the lead connecting the transformer to the burden as part of the voltage on the transformer secondary winding to be measured.

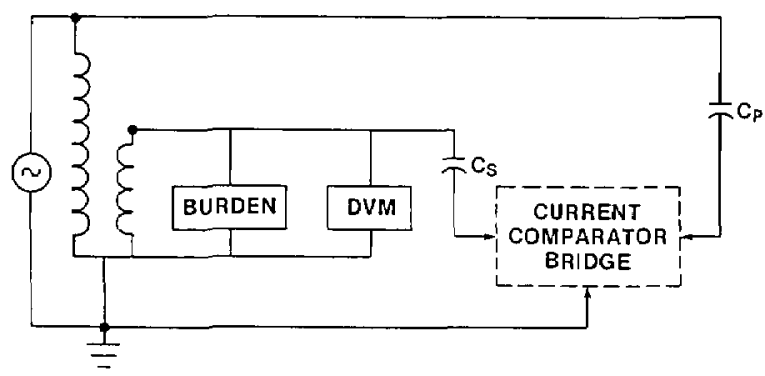

Figure 6. Measurement circuit for the calibration of a voltage transformer. Connection of low-voltage capacitor as shown is incorrect. 
Table 3. ANSI standard burdens

\begin{tabular}{ccc}
\hline \hline ANSI burden & Volt-amperes & Power factor (lagging) \\
\hline W & 12.5 & 0.10 \\
X & 25 & 0.70 \\
M & 35 & 0.20 \\
Y & 7 & 0.85 \\
Z & 200 & 0.85 \\
ZZ & 400 & 0.85 \\
\hline
\end{tabular}

Another major concern in the measurement of the ratio and phase angle of a voltage transformer is the proper definition of the ground point and the avoidance of ground loops. This can best be illustrated by a few examples. In figure 7 , some common mistakes are shown. The transformer is energized in such a manner that significant current is forced to flow between the transformer ground and the circuit ground. The resulting voltage drop in the lead connecting the transformer and ground will be part of the ratio and phase angle measured. The high-voltage capacitor is not connected directly to the primary of the transformer under test. The measurement of the ratio and phase angle, therefore, includes the effect of the voltage drop in the lead between the point where the capacitor is connected to the power source and the transformer. In addition, as there are three different "ground" points in the circuit and it is not, in general, possible to know the voltages and impedances between these points, a measurement error is probable.

In figure 8 the problem has been eliminated by defining the low-voltage terminal of the transformer as ground. Although this point may significantly differ from the building or utility ground, from the measurement point of view this is the correct ground. It is important that the shields of the three-terminal capacitors, the bridge detector ground, and all other measurement grounds each be connected directly to this point.

In figure 5 the preferred method of wiring a voltage transformer calibration circuit is shown. The client's transformer is connected in such a way that the energizing current does not flow between the transformer and the measurement ground. All measurement grounds are connected to the transformer ground point. The two capacitors are connected directly to the primary and secondary terminals of the transformer. Only one ground is used in the circuit. While it is not always possible to connect the transformer as in figure 5 , this is the best choice. Otherwise tests are required to ensure that systematic errors are not compromising the measurement results.

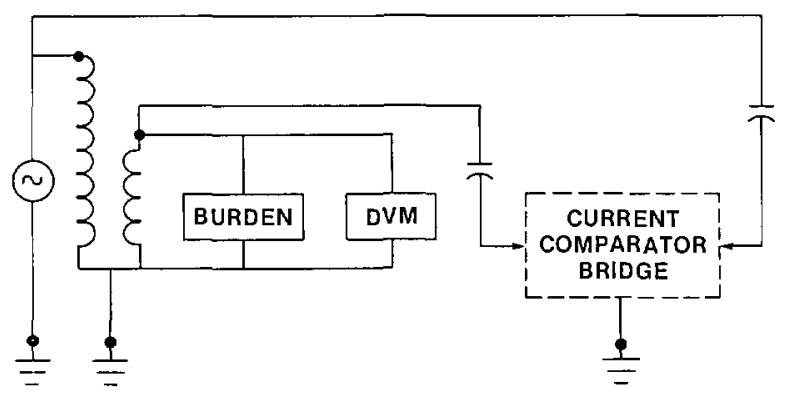

Figure 7. Measurement circuit for the calibration of a voltage transformer. Grounds are poorly defined.

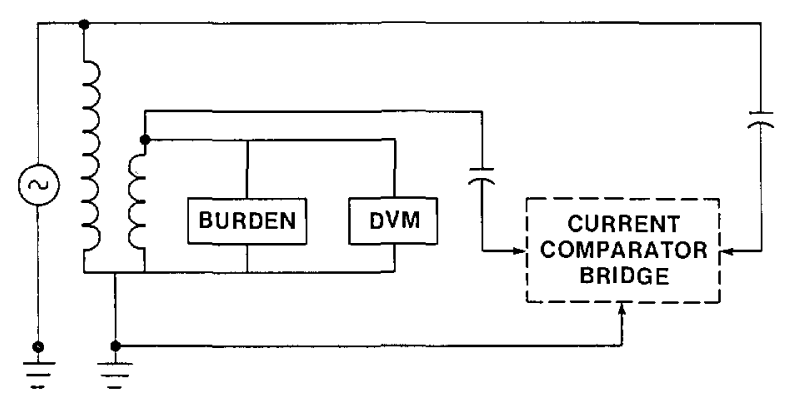

Figure 8. Measurement circuit for the calibration of a voltage transformer. Measurement ground is defined. Transformer excitation current flows from the measurement ground to building ground.

3.3.5 Burdens The burden attached to the secondary of the client's transformer (as shown in fig. 5 ) is specified by the client. In general this would not be the burden corresponding to the maximum volt-ampere rating of the transformer but instead would be equal to the burden attached to the transformer in its intended use. For example, if the transformer will only have a digital voltmeter attached to its secondary, a calibration with a secondary impedance of one megohm would be more useful than one with an ANSI ZZ burden attached. Since the ANSI burdens are often requested, they are summarized in table 3 [7]. By convention these burdens are defined for a frequency of $60 \mathrm{~Hz}$ only. 
3.3.6 Substitute Burdens If the client of the service does not send the secondary burden with the transformer, the National Institute of Standards and Technology will provide the burden. It is not practical to have available and adequately characterized all of the anticipated burdens. Fortunately this is not necessary. If the ratio and phase angle of a transformer is known for two different burden values, the ratio and phase angle at any other burden can be calculated (with certain limitations) [8]. A derivation of the formulas relating the ratios and phase angles at zero and some other known burden value are given in the appendix and presented in abbreviated form below.

The voltage transformer will be represented as an ideal transformer with some unknown series output impedance $Z_{0}$, as shown in figure 9. The model has been shown to be sufficiently accurate experimentally. The relationship between the input voltage $E_{\mathrm{i}}$, and the output voltage with zero burden $E_{0}$, is:

$$
\frac{E_{\mathrm{i}}}{E_{0}}=N R C F_{0} e^{-j \Gamma_{0}}=\left|\frac{E_{\mathrm{i}}}{E_{0}}\right| e^{-j \Gamma_{0}},
$$

where $N$ is the nominal (or turns) ratio of the transformer, $R C F$ is the ratio-correction factor $\left(N \times R C F=\right.$ actual ratio) at zero burden, $\Gamma_{0}$ is the angle by which the secondary voltage vector leads the primary voltage vector and $j=\sqrt{-1}$. A similar relationship exists between the input voltage $E_{\mathrm{j}}$, and the output voltage $E_{\mathrm{c}}$, with secondary burden $C$ (having impedance $Z_{\mathrm{c}}$ ) shown in figure 10 :

$$
\frac{E_{\mathrm{i}}}{E_{\mathrm{c}}}=N R C F_{\mathrm{c}} e^{-j \Gamma_{\mathrm{c}}},
$$

where $R C F_{\mathrm{c}}$ is the ratio correction factor with secondary burden $C$ and $\Gamma_{\mathrm{c}}$ is the corresponding phase angle. If the transformer is measured at zero burden $\left(R C F_{0}\right.$ and $\left.\Gamma_{0}\right)$ and at burden $T\left(R C F_{t}\right.$ and $\left.\Gamma_{t}\right)$, the ratio correction factor and phase angle at burden $C$ are approximately given by:

$$
\begin{aligned}
R C F_{\mathrm{c}} \approx & R C F_{0}+\frac{B_{\mathrm{c}}}{B_{\mathrm{t}}}\left[\left(R C F_{\mathrm{t}}-R C F_{0}\right)\right. \\
& \cos \left(\theta_{\mathrm{t}}-\theta_{\mathrm{c}}\right)+\left(\Gamma_{\mathrm{t}}-\Gamma_{\mathrm{o}}\right) \\
& \left.\sin \left(\theta_{\mathrm{t}}-\theta_{0}\right)\right]
\end{aligned}
$$

where $B_{\mathrm{c}}=1 / Z_{\mathrm{c}}$ is the burden in $\Omega^{-1}$ of the impedance $Z_{\mathrm{c}}$, and

$$
\begin{aligned}
\Gamma_{\mathrm{c}} \approx & \Gamma_{\mathrm{o}}+\frac{B_{\mathrm{c}}}{B_{\mathrm{t}}}\left[\left(\Gamma_{\mathrm{t}}-\Gamma_{\mathrm{o}}\right) \cos \left(\theta_{\mathrm{t}}-\theta_{\mathrm{c}}\right)-\left(R C F_{\mathrm{t}}-R C F_{0}\right)\right. \\
& \left.\sin \left(\theta_{\mathrm{t}}-\theta_{\mathrm{c}}\right)\right] .
\end{aligned}
$$

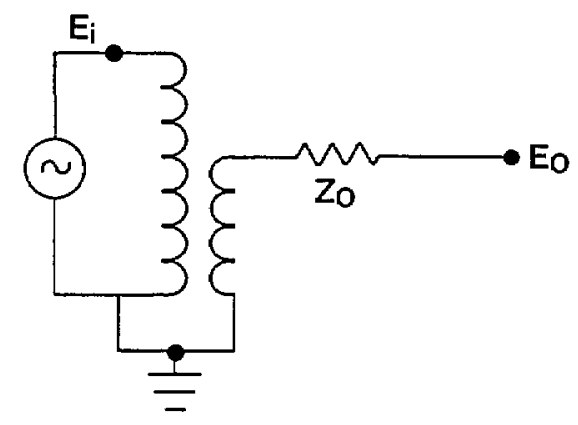

Figure 9. Equivalent circuit of a voltage transformer.

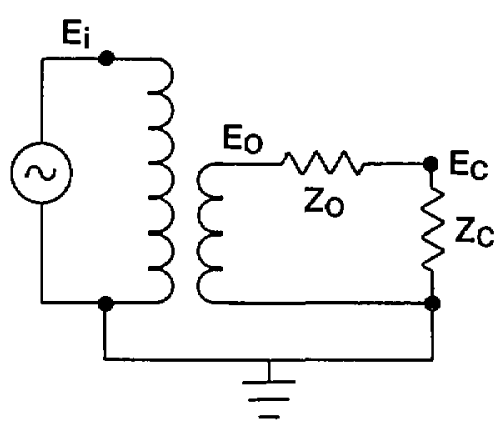

Figure 10. Equivalent circuit of a voltage transformer with secondary burden $Z_{\text {c. }}$.

The power factor of burden $C$ is $\cos \theta_{\mathrm{c}}, R C F_{\mathrm{c}}$ is the ratio correction factor calculated for burden $C$, and $\Gamma_{\mathrm{c}}$ is the angle by which the secondary voltage leads the primary voltage for burden $C$.

Equations (7) and (8) can be used to calculate the $R C F$ and phase angle for some secondary burden, $C$, if the ratio correction factors and phase angles are known at some other burden $T$, and at zero burden. In practice, at NIST, capacitive burdens are used for the " $T$ " or known burdens in eqs (7) and (8). The main reason is their stability. The heat generated in a large resistive burden, for example, is likely to cause the burden's impedance value to vary. Capacitors, in addition, are compact so even the $\mathrm{ZZ}$ burden in table 3 is easy to handle. At NIST, capacitive burden boxes have been constructed in a binary layout (fig. 11) so that capacitors from 1 to $32 \mu \mathrm{F}$ can be switched in and out allowing any capacitance value from zero to $63 \mu \mathrm{F}$. Since a $\mathrm{ZZ}$ burden is equivalent to a $74 \mu \mathrm{F}$ capacitor at $120 \mathrm{~V}$, two such burden boxes are sufficient for nearly all the calibrations at NIST.

Several approximations were made to derive eqs (7) and (8). The approximations relate to the relative ratio of the transformer's output impedance $Z_{0}$ to the impedance of the secondary burden $Z_{\mathrm{t}}$ or $Z_{\mathrm{c}}$. 
The smaller this ratio, the more accurate are eqs ( 7$)$ and (8). This ratio also affects the differences, $R C F_{t}-R C F_{0}$ and $\Gamma_{1}-\Gamma_{0}$. If the ratio correction factor difference is 0.001 or less, and if the phase angle difference is $1 \mathrm{mrad}$ or less than eqs (7) and (8) should be accurate to within $\pm 10 \mathrm{ppm}$ for the ratio correction factor and to within $\pm 10 \mu \mathrm{rad}$ for the phase angle if it is assumed that the ratio of the burdens is known with no more than \pm 1 percent uncertainty. Data over the years has indicated that eqs (7) and (8) are always at least that accurate. In order to identify any problems, an extra measurement is made at a different secondary burden to test the predictive capabilities of eqs (7) and (8) for the transformer under test. If a problem is discovered, the error budget is adjusted accordingly.

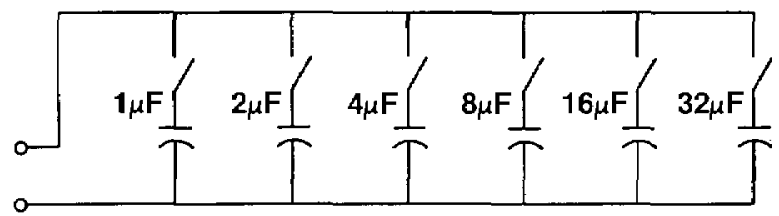

Figure 11. Capacitive burden box.

The above discussion might enable clients of the voltage transformer calibration service to better design their calibration requests. Using eqs (7) and (8), the client might be able to reduce the number of measurements required. A note of caution is in order. It is likely that using a zero burden result and a 10 volt-ampere burden result to predict the transformer's behavior at a ZZ burden may lead to large inaccuracies. The reasons are twofold. First, the differences $R C F_{\mathrm{t}}-R C F_{0}$ and $\Gamma_{\mathrm{t}}-\Gamma_{0}$ are likely to be small for a burden as small as 10 volt-amperes and extrapolations can cause large errors. The second reason can be seen from figure 10. The higher current of the $\mathrm{ZZ}$ burden will cause $Z_{0}$ to heat up and increase in value, leading to errors if eqs (7) and (8) are used. Somewhat better results are likely if one uses a $\mathrm{ZZ}$ burden result to predict a transformer's behavior at 10 volt-amperes. However, it is best to choose burden $T$ to have a volt-ampere rating the same order of magnitude as the burden of interest $C$. Also, the values in eqs (7) and (8) are all to be measured at the same frequency and at the same secondary voltage.

3.3.7 Harmonic Effects The measurement of the ratio and phase angle of a voltage transformer can be affected by the presence of harmonics in the voltage waveform. If a tuned null detector is not used, the balance of a bridge circuit can be difficult in the presence of harmonics and often a precise balance is not possible resulting in increased measurement uncertainties. Harmonics can also lead to errors in measuring the magnitude of the secondary voltage. For example, if an average reading, rms scaled voltmeter measured a $100-\mathrm{V}$ rms fundamental with an in-phase 3-V rms third harmonic, the meter would read $101 \mathrm{~V}$. Setting the voltage to read $100 \mathrm{~V}$ on the meter would result in a $1-\mathrm{V}$ discrepancy between the intended and actual voltage. Many transformers have large enough voltage coefficients for this $1-V$ error in the voltage setting to have a non-negligible effect on the measured ratio correction factor and phase angle. If instead, a true rms voltmeter were used to measure this signal, the measured voltage would be $100.045 \mathrm{~V}$ and the resulting error would be negligible. At NIST three different steps are taken to lessen the effects of harmonics. The first is to try to minimize the harmonic content of the power supply. The supply used for most of the calibrations has a total harmonic distortion of order $0.2 \%$ of the fundamental. Second, a tuned detector is used to assure that the balance conditions are for the fundamental component of the voltage waveform. And third, all voltage measurements are made with true-rms voltmeters.

3.3.8 Voltage Dependence of Standard Capacitor An additional measurement concern is the voltage coefficient of the high-voltage standard capacitor shown in figure 5. Although no absolute measurements are required to calibrate a voltage transformer, the ratio of the two standard capacitors must be known. The problem is that the lowvoltage standard capacitor typically has a maximum voltage rating of $500 \mathrm{~V}$, and both the primary of the transformer and the high-voltage standard capacitor might be energized to $100 \mathrm{kV}$. Since the capacitor ratio measurement must be done at less than $500 \mathrm{~V}$, the voltage dependence of the high-voltage capacitor is important. This problem was discussed in section 3.2 .

\section{Measurement Instrumentation}

The calibration of voltage transformers and high-voltage capacitors at NIST requires the combined use of standard capacitors and the current comparator bridge. Standard capacitors have been thoroughly discussed in the literature $[5,6,9$,$] . The$ care that must be taken with their use in these types of measurements has been discussed above. The current comparator bridge will be discussed in this section. 
The current comparator bridge can be thought of as a voltage comparator transformer arm bridge in which the detector and power source have been interchanged. Traditionally, the disadvantage of the current comparator bridge versus the voltage comparator bridge is the signal-to-noise level. For high-voltage measurement applications, this is no longer a problem. Kusters and Petersons were the first to develop this bridge for the comparison of two capacitors at high voltage [3]. A basic current comparator bridge is shown in figure 12 . The current in the unknown capacitor, $C_{x}$, is balanced against the current in the standard capacitor, $C_{\mathrm{s}}$, by varying the turns ratios, $N_{\mathrm{s}}$ and $N_{\mathrm{x}}$.

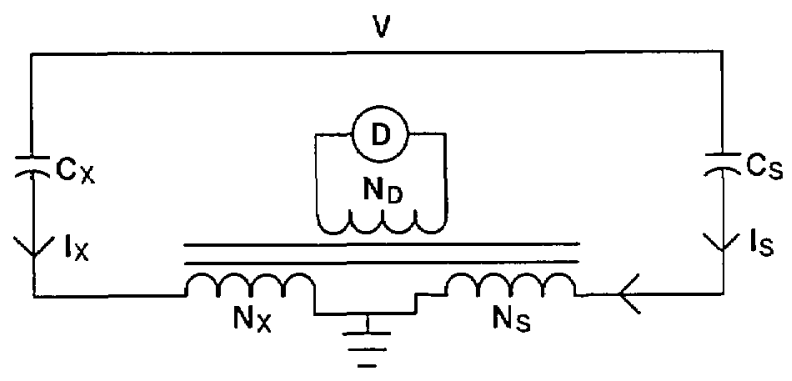

Figure 12. Basic current comparator bridge.

Balance is achieved when the signal at the detector $\mathrm{D}$ is equal to zero. At balance $I_{\mathrm{x}} N_{\mathrm{x}}=I_{\mathrm{s}} N_{\mathrm{s}}$ or:

$$
V 2 \pi f C_{\mathrm{x}} N_{\mathrm{x}}=V 2 \pi f C_{\mathrm{s}} N_{\mathrm{s}},
$$

where $f$ is the frequency. This balance equation can also be expressed as:

$$
C_{x}=\frac{N_{\mathrm{s}}}{N_{x}} C_{\mathrm{s}}
$$

The bridge shown in figure 12 has no means of balancing the in-phase current resulting from a non-ideal unknown capacitor $C_{\mathrm{x}}$. The current comparator in figure 13 does have the capability of balancing both the in-phase and quadrature components of the capacitive current. The difficulty with the approach used in figure 13 is that the applied high voltage is across the variable resistance $R_{\mathrm{s}}$. It is nearly impossible to design a stable high-voltage variable resistor with negligible phase angle. Another means is necessary to balance the in-phase current, preferably at low voltage using well-characterized components.

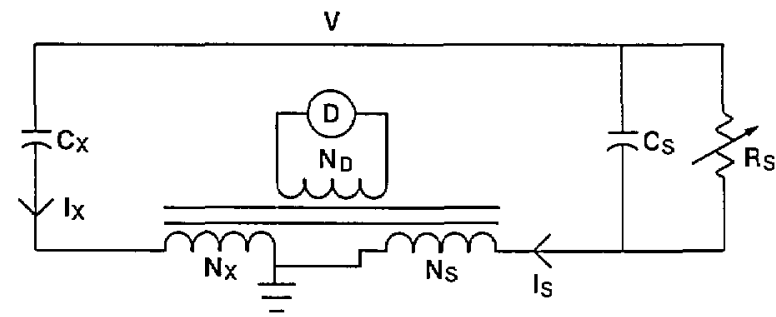

Figure 13. Current comparator bridge with high-voltage resistor for in-phase current balance.

The current comparator shown in figure 14 provides a satisfactory means of achieving both the in-phase and quadrature current balances. The quadrature current balance is identical to that in figures 12 and 13 above. The in-phase current balance is accomplished at low voltage with the aid of an operational amplifier. The current from the standard capacitor, after passing through the $N_{\mathrm{s}}$ winding, goes to the inverting input of the operational amplifier. This point is at virtual ground so the capacitive current balance, eq (10), is not affected. The feedback capacitor $C_{\mathrm{r}}$ causes the output voltage of the operational amplifier to be a small fraction $\left(C_{s} / C_{\mathrm{f}}\right.$ where $C_{\mathrm{f}}$ is approximately $\left.10 \mu \mathrm{F}\right)$ of the applied voltage and $\pi$ radians out of phase with it. The inductive voltage divider allows a known fraction, $\alpha$, of this output signal to be applied across a standard resistor $R$. As can be seen from figure 14, the signal is first inverted before the resistor in order to have the correct phase relationship with the unknown in-phase current.

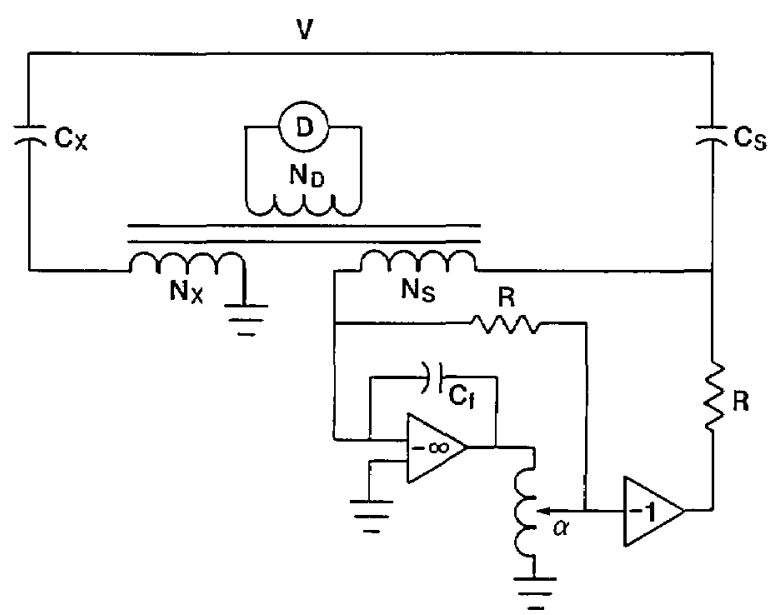

Figure 14. Current comparator with superior in-phase current balance. 
It is necessary that the non-inverted signal be applied to an identical standard resistor as shown in figure 14 so that the current from the standard winding $N_{\mathrm{s}}$ reaching the operational amplifier has no phase defect. The in-phase current into the standard winding $N_{\mathrm{s}}$ is then equal to:

$$
I_{\text {in }}=\frac{\left(\alpha V C_{\mathrm{s}} / C_{\mathrm{f}}\right)}{R}
$$

Since the quadrature current $I_{\text {out }}=V 2 \pi f C_{\mathrm{s}}$, the dissipation factor is:

$$
\mathrm{DF}=\frac{I_{\text {in }}}{I_{\text {out }}}=\frac{\alpha V C_{\mathrm{s}}}{2 \pi f R V C_{\mathrm{f}} C_{\mathrm{s}}}
$$

or

$$
\mathrm{DF}=\frac{\alpha}{2 \pi f R C_{\mathrm{f}}}
$$

The resistor $R$ can be chosen so that $\alpha$ is direct reading in percent or milliradians.

In some cases, particularly for larger capacitors, it is necessary to make a four-terminal measurement. This is required when the lead and winding impedances become a significant fraction of the impedance to be measured. Figure 15 shows a current comparator bridge with this capability. Because of the non-negligible lead and winding impedance, there is some voltage $e$ at the lowvoltage terminal of the capacitor. This voltage signal is inverted as shown in figure 15 and connected to the $N_{\mathrm{s}}$ winding through a capacitor $\mathrm{C}_{\mathrm{s}^{\prime}}$. The current through the unknown capacitor is:

$$
I_{\mathrm{x}}=j 2 \pi f(V-e) C_{\mathrm{x}} .
$$

The current reaching the $\mathrm{N}_{\mathrm{s}}$ winding is:

$$
I_{\mathrm{s}}=j 2 \pi V C_{\mathrm{s}}-j 2 \pi f e C_{\mathrm{s}^{\prime}}
$$

If $C_{\mathrm{s}^{\prime}}$ is adjusted prior to the measurement to be equal to $C_{\mathrm{s}}$ then eq (15) reduces to:

$$
I_{\mathrm{s}}=j 2 \pi f(V-e) C_{\mathrm{s}} .
$$

Comparing this with eq (14), the effect of the compensation circuit has been to place the same voltage across both the standard and unknown capacitors. This is exactly what is required for lead compensation.

Figure 16 shows the last enhancement of the bridge to be discussed. The National Institute of Standards and Technology's current comparator bridge has an internal range of 1000:1 (i.e., the maximum value of $N_{\mathrm{d}} / N_{\mathrm{x}}$ is 1000 ). The external current transformer shown in figure 16 , referred to as a range extender, increases the measurement range by a factor of 1000 allowing the comparison of two currents differing in magnitude by as much as a factor of a million. As with the transformers internal to the current comparator bridge, the accuracy requirements on the range extender are quite stringent. Further details on the design of a ppm current comparator and the specifics of NIST's current comparator bridge are available in the literature $[10,11]$.

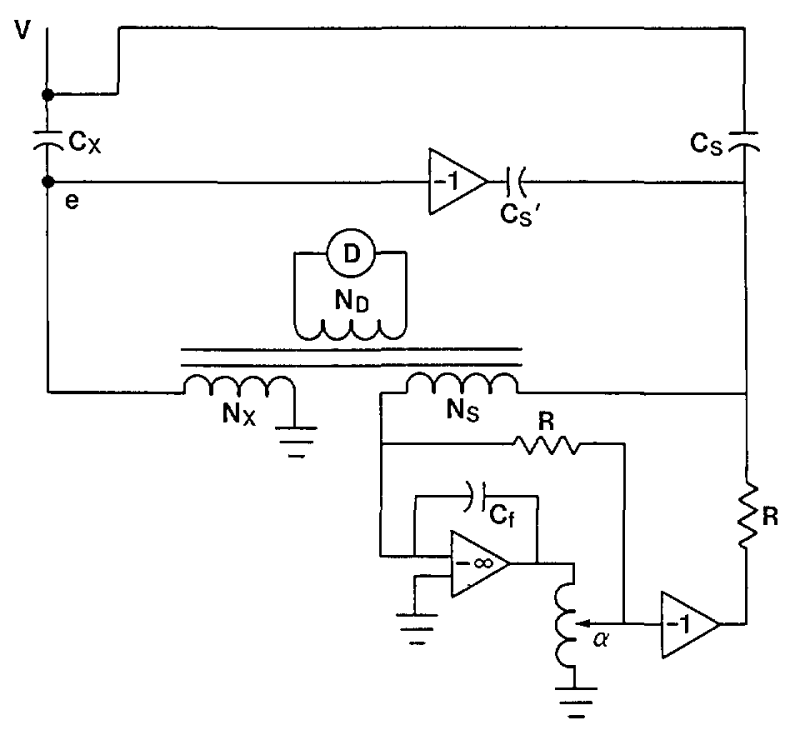

Figure 15. Current comparator bridge modified for fourterminal capacitance measurements.

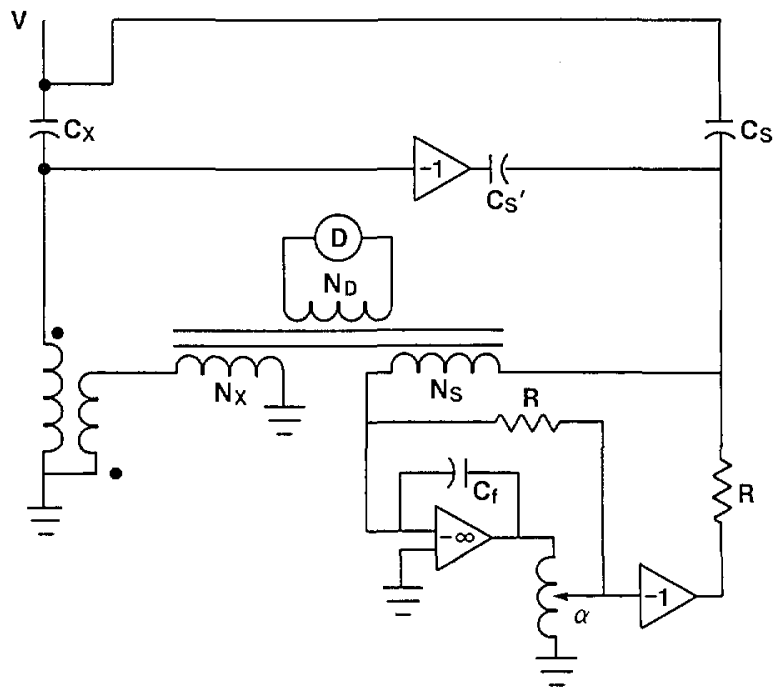

Figure 16. Current comparator bridge with external range extender. 
The current comparator bridge is quite straightforward to use and has proven to be rugged in practice. In order to monitor the behavior of NIST's current comparator bridge, a check standard is maintained. In this case, the check standard consists of two high quality standard capacitors. The ratio of the two capacitors is measured quarterly. For the last 8 years, this ratio has been stable to within about $20 \mathrm{ppm}$ as can be seen in table 4 .

Table 4. Check standard history

\begin{tabular}{rc||rc}
\hline \hline Date & Capacitance ratio & \multicolumn{1}{|c}{ Date } & Capacitance ratio \\
\cline { 3 - 4 } $6 / 80$ & 1.000025 & $10 / 84$ & 1.000032 \\
$6 / 81$ & 1.000028 & $4 / 85$ & 1.000041 \\
$9 / 81$ & 1.000027 & $6 / 85$ & 1.000042 \\
$1 / 82$ & 1.000027 & $10 / 85$ & 1.000041 \\
$4 / 82$ & 1.000026 & $12 / 85$ & 1.000042 \\
$7 / 82$ & 1.000026 & $1 / 86$ & 1.000041 \\
$9 / 82$ & 1.000028 & $5 / 86$ & 1.000044 \\
$1 / 83$ & 1.000030 & $7 / 86$ & 1.000044 \\
$3 / 83$ & 1.000031 & $10 / 86$ & 1.000044 \\
$6 / 83$ & 1.000033 & $2 / 87$ & 1.000044 \\
$8 / 83$ & 1.000031 & $7 / 87$ & 1.000046 \\
$12 / 83$ & 1.000031 & $12 / 87$ & 1.000044 \\
$1 / 84$ & 1.000032 & $4 / 88$ & 1.000040 \\
$5 / 84$ & 1.000033 & $11 / 88$ & 1.000046 \\
\hline
\end{tabular}

The drift can readily be attributed to the two capacitors. The $9 \mathrm{ppm}$ change between 10/84 and $4 / 85$ occurred apparently after one of the capacitors had been used for another purpose. An independent measurement of that capacitor verified the change. While the use of this check standard cannot prove that the bridge is still working to the ppm level, it can alert the user of changes large enough to affect calibration results. Of course, since the two capacitive currents are largely balanced using stable passive components (i.e., transformer windings), one expects that the bridge should be stable. It should be noted that if a transformer winding were to become open or short circuited the result would be dramatic and readily observed by the operator.

The situation with the dissipation factor (or inphase current) balance is different as active components play an important role. Also, it is difficult to design a stable dissipation factor standard to act as a check standard. This problem has been overcome by using the circuit in figure 17. Standard capacitors are connected to the standard and unknown sides of the bridge. The known in-phase current is applied with the use of the inductive voltage divider and a resistor as shown. The advantage of this circuit is that the voltage across the resistor is small $(\sim 0.3 \mathrm{~V})$. However, because of the small voltage, any error voltage, $\epsilon$, at the low side of the resistor, $R$, becomes important. The in-phase current entering the $N_{x}$ winding is:

$$
I_{\text {in }}=\frac{a V-\epsilon}{R},
$$

where $\alpha$ is the ratio of the inductive voltage divider $(\alpha<1)$. The dissipation factor $I_{\text {in }} / I_{\text {out }}$ is then equal to:

$$
D F=\left[\frac{a V-\epsilon}{(V-\epsilon) R 2 \pi f C_{\mathrm{x}}}\right]
$$

The effect of $\epsilon$ can be significant at the ppm level and needs to be eliminated. The circuit in figure 18 is identical to that in figure 17 except that the input of the inductive voltage divider is grounded. The dissipation factor in this case is then:

$$
D F_{0}=\left[\frac{-\epsilon}{(V-\epsilon) R 2 \pi f C_{\mathrm{x}}}\right]
$$

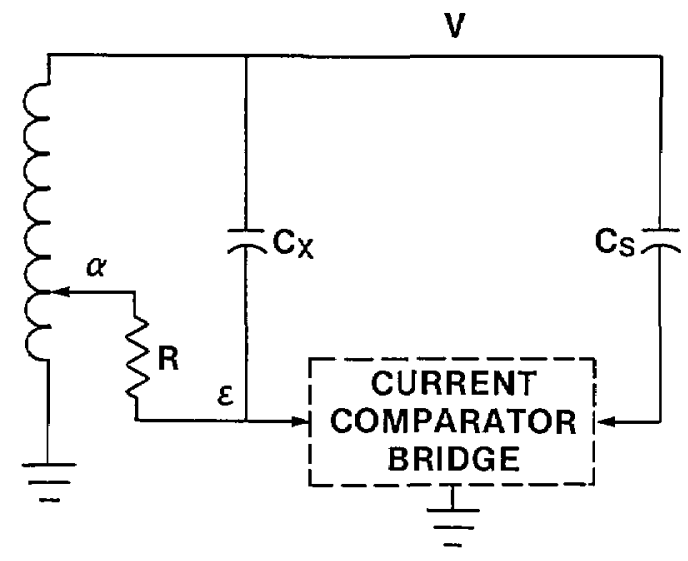

Figure 17. Circuit for checking operation of dissipation factor measurement of current comparator bridge.

Since $\epsilon \ll V$ subtracting eq (19) from eq (18) one obtains:

$$
D F_{\mathrm{m}}=D F-D F_{0}=\frac{a}{2 \pi f R C_{\mathrm{x}}} .
$$




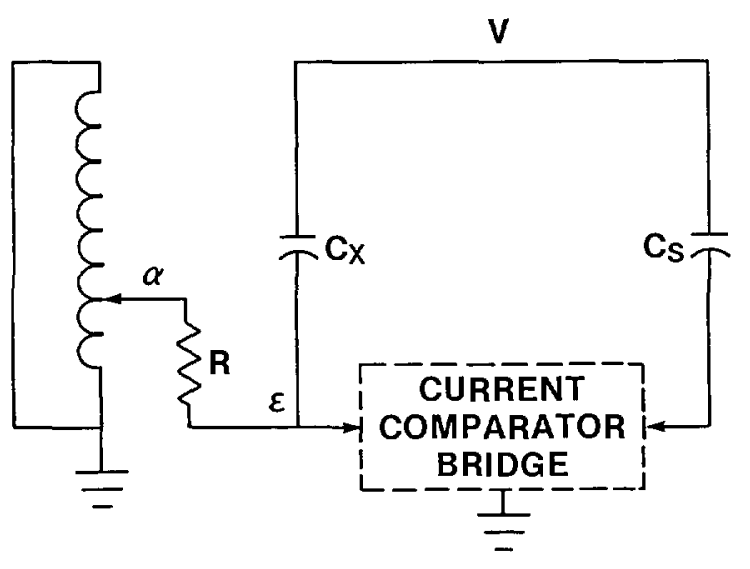

Figure 18. Circuit for checking operation of dissipation factor measurement of current comparator bridge. Input is grounded in order to measure $\epsilon$ in eq (18).

At NIST typical values of $\alpha$ are $0.003,0.0003$, $-0.0003,-0.003$. With a $1 \mathrm{M} \Omega$ resistor and a 1000 -pF standard capacitor this enables a near full scale test of the dissipation factor on its four ranges. Recent results are shown in table 5. The dissipation factor values are all in units of percent.

Agreement between the calculated values in eq (20) and the corrected measurement $D F_{\mathrm{m}}$ (the last two columns) are well within $\pm 0.2 \%$ of the measured value. This check is performed at approximately 6-month intervals.

It is further proposed that an additional check standard be obtained and measured quarterly. Specifically, a voltage transformer measured regularly at a ratio of 10:1 would give an additional check on the phase angle circuitry and on the bridge windings at something other than a 1:1 ratio.

\section{Measurement Uncertainties \\ 5.1 Voltage Transformers}

The records of the National Institute of Standards and Technology show examples of voltage transformers that have been calibrated at 5-year intervals over a period of 30 to $\mathbf{4 0}$ years. Invariably the original uncertainty statement covers any variation in ratio correction factor and phase angle observed over this period of time. Voltage transformers are often used by the client in conjunction with other equipment to measure some quantity. For example, used with a current transformer and watthour meter, a voltage transformer can help provide a measure of the energy consumed by a large power transformer. Thus it is important to the clients of this calibration service to
Table 5. Dissipation factor check standard

\begin{tabular}{|c|c|c|c|c|c|}
\hline Date & $\alpha$ & $\begin{array}{l}\text { Measured } \\
(D F)\end{array}$ & $\begin{array}{l}\text { Correction } \\
\left(D F_{0}\right)\end{array}$ & $\begin{array}{c}\text { Corrected } \\
\left(D F_{\mathrm{m}}\right)\end{array}$ & $\begin{array}{l}\text { Theoretical } \\
\left(\alpha / 2 \pi f R C_{\mathrm{x}}\right)\end{array}$ \\
\hline \multirow[t]{4}{*}{$7 / 82$} & 0.0003 & 0.08003 & & 0.07983 & 0.07977 \\
\hline & 0.003 & 0.7982 & .0002 & 0.7980 & 0.7977 \\
\hline & -0.003 & -0.7979 & .0002 & -0.7981 & -0.7977 \\
\hline & -0.0003 & -0.07959 & 0.0002 & -0.07979 & -0.07977 \\
\hline \multirow[t]{4}{*}{$3 / 83$} & 0.0003 & 0.08135 & -0.00014 & 0.08149 & 0.08147 \\
\hline & 0.003 & 0.81455 & & & 0.8147 \\
\hline & -0.003 & -0.81475 & -0.00015 & -0.8 & -0.8147 \\
\hline & -0.0003 & -0.08160 & & -0.08146 & -0.08147 \\
\hline \multirow[t]{4}{*}{$10 / 83$} & 0.0003 & 0.07952 & -0.0001 & 0.07962 & 0.07959 \\
\hline & 0.003 & 0.75 & -0.0 & & 0.7959 \\
\hline & -0.003 & -0.79 & -0.0 & -0.7 & -0.7959 \\
\hline & -0.0003 & -0.07965 & -0.00 & -0.0 & -0.07959 \\
\hline \multirow[t]{4}{*}{$1 / 84$} & 0.0003 & 0.08174 & 0.00029 & 0.08145 & 0.08143 \\
\hline & 0.003 & 0.8 & & & 0.8143 \\
\hline & -0.003 & -0.8 & 0.00 & -0.8 & -0.8143 \\
\hline & -0.0003 & -0.08110 & 0.00029 & -0.08139 & -0.08143 \\
\hline \multirow[t]{4}{*}{$5 / 84$} & 0.0003 & 0.08 & & & 0.08071 \\
\hline & 0.003 & 0.8 & & & 0.8071 \\
\hline & -0.003 & -0.8 & 0.0 & -0 & -0.8071 \\
\hline & -0.0003 & -0.08050 & 0.0002 & -0 . & -0.08071 \\
\hline \multirow[t]{4}{*}{$11 / 84$} & 0.0003 & 0.08000 & 0.0000 & 0.08000 & 0.07997 \\
\hline & 0.003 & 0.8 & & & \\
\hline & -0.003 & -0.8000 & 0.0000 & -0.8000 & -0.7997 \\
\hline & -0.0003 & -0.08000 & 0.00 & -0.08000 & -0.07997 \\
\hline \multirow[t]{4}{*}{$4 / 85$} & 0.0003 & & & & 0.08059 \\
\hline & 0.003 & & & & \\
\hline & -0.003 & -0.8 & & -0 . & -0.80 \\
\hline & -0.0003 & -0.08050 & 0.0000 & -0.08 & -0.0805 \\
\hline \multirow[t]{4}{*}{$12 / 85$} & 0.0003 & 0.08070 & -0.0001 & & 0.08071 \\
\hline & 0.003 & & & & 0.8071 \\
\hline & -0.003 & -0.80 & & & -0.8071 \\
\hline & -0.0003 & -0.08070 & & & -0.08071 \\
\hline \multirow[t]{4}{*}{$11 / 86$} & 0.0003 & & & & \\
\hline & 0.003 & & & & 0.8046 \\
\hline & -0.003 & -0.8 & -0.0 & -0 & -0.8046 \\
\hline & -0.0003 & -0.08067 & & & -0.0804 \\
\hline \multirow[t]{4}{*}{$7 / 87$} & 0.0003 & 0.08031 & -0.0002 & 0.08051 & 0.08045 \\
\hline & 0.003 & & & & 0.8045 \\
\hline & -0.003 & -0.80 & -0.00 & -0.8 & -0.8045 \\
\hline & -0.0003 & -0.08071 & & -0.08051 & -0.08045 \\
\hline \multirow[t]{4}{*}{$12 / 87$} & 0.0003 & & & & 0.08 \\
\hline & 0.003 & & & & 0.8039 \\
\hline & -0.003 & -0.80 & 0.00 & -0 . & -0.8039 \\
\hline & -0.0003 & & 0.0001 & & \\
\hline \multirow[t]{4}{*}{$8 / 88$} & 0.0003 & 0.08010 & -0.0003 & 0.08040 & 0.08030 \\
\hline & 0.003 & 0.8037 & -0.0003 & 0.8040 & 0.8030 \\
\hline & -0.003 & -0.8043 & -0.0003 & -0.8040 & -0.8030 \\
\hline & -0.0003 & -0.08070 & -0.0003 & -0.08040 & -0.08030 \\
\hline \multirow[t]{4}{*}{$11 / 88$} & 0.0003 & 0.08 & & & 0.08128 \\
\hline & 0.003 & & & & 0.8128 \\
\hline & -0.003 & -0.8140 & 0.0000 & -0.8140 & -0.8128 \\
\hline & -0.0003 & -0.08135 & 0.0000 & -0.08135 & -0.08128 \\
\hline
\end{tabular}


obtain a meaningful uncertainty statement that reflects the contribution the voltage transformer would make to their total error budget.

As mentioned earlier in this paper, voltage transformers calibrated at NIST generally fall into two accuracy classes: $\pm 0.03 \%$ uncertainty for ratio correction factor, $\pm 0.3 \mathrm{mrad}$ for phase angle; and $\pm 0.01 \%$ for ratio correction factor, $\pm 0.1 \mathrm{mrad}$ for phase angle. While it would be possible in some cases to report smaller uncertainties to the clients by more thorough determinations of such parameters as voltage coefficients, proximity effects, and burden dependencies, the present service provides an economical way to present meaningful error statements to the clients and meets their needs.

The analysis of the uncertainties for the ratio correction factor measurements are summarized in table 6. The units are in ppm. The values in parentheses apply to the higher accuracy voltage transformers described in section 2.1. The uncertainties for the phase angle measurement of voltage transformers are the same as is shown in table 6 except the units are microradians instead of ppm.

Table 6. Contributions to uncertainty

\begin{tabular}{lcc}
\hline \hline & \multicolumn{2}{c}{ Uncertainties } \\
& Random & Systematic \\
\hline Bridge measurement & $\pm 2( \pm 2)$ & $\pm 75( \pm 25)$ \\
Secondary voltage setting & & $\pm 50( \pm 10)$ \\
Burden setting & & $\pm 50( \pm 10)$ \\
Transformer self-heating & & $\pm 75( \pm 20)$ \\
Capacitance ratio measurement & $\pm 2( \pm 2)$ & $\pm 5( \pm 5)$ \\
\hline
\end{tabular}

To calculate the uncertainties reported to the client, the systematic uncertainties tabulated above are algebraically summed and added to three times the root sum of squares of the random uncertainties. The results are shown in table 7 .

Table 7. Total estimated uncertainties

\begin{tabular}{lll}
\hline \hline Ratio correction factor & $\pm 0.03 \%$ & $( \pm 0.01 \%)$ \\
Phase Angle & $\pm 0.3 \mathrm{mrad}$ & $( \pm 0.1 \mathrm{mrad})$ \\
\hline
\end{tabular}

The values in table 6 are approximate. Some transformers demonstrate stronger voltage dependences than others or stronger burden dependences. In some cases the values in table 7 must be adjusted for such transformers. The purpose of the above tables is to give the users an idea of the sources of errors and how they are used to calculate an uncertainty statement.
Since most of the sources of uncertainty presented in table 6 originate from the transformer under test, NIST could in principle measure a nearly ideal voltage transformer to much better accuracy than shown in table 7. Such a test would be expensive because of the time-consuming care that would be required.

\subsection{Capacitors}

The National Institute of Standards and Technology has the capability to measure the ratio of two capacitors to an estimated systematic uncertainty of $\pm 1 \mathrm{ppm}$ and $\pm 1 \times 10^{-6} \pm 1 \%$ of the measured value for the relative dissipation factor. The values of the standard capacitors used for these comparisons are known to $\pm 10 \mathrm{ppm}$ for capacitance $\left( \pm 1 \times 10^{-6}\right.$ for dissipation factor). The random uncertainty associated with the capacitance measurement is $\pm 1 \mathrm{ppm}$ and $\pm 1 \times 10^{-6}$ for dissipation factor. Conservatively then, NIST could calibrate a client's capacitor to an overall uncertainty of $\pm 15 \mathrm{ppm}$ in capacitance and $\pm 5 \times 10^{-6} \pm 1 \%$ of the value for dissipation factor. In general, the quoted uncertainty is always larger than this except for low-voltage standard capacitors similar to those used at the National Institute of Standards and Technology. (Low-voltage standard capacitors are in general calibrated elsewhere at NIST. The service described here provides higher voltage calibration of these same capacitors.)

The uncertainty statements for high-voltage standard capacitors and power-factor capacitors depend on the stability of these devices during the course of the NIST measurements. The stability is influenced by both the voltage dependence of the device and self-heating (i.e., the capacitance and dissipation factors vary as the internal energy dissipated heats the device). Self-heating effects are more important for power-factor capacitors. Some power-factor capacitors demonstrate significant hysteresis effects. Assigning an uncertainty statement to these measurements depends on the specific behavior of the capacitor. If self-heating is a problem the calibration report clearly must specify the amount of time the capacitor was energized before the measurement was made. If hysteresis effects are detected they are so noted. Because of the nature of most of these devices, the calibration reports for capacitors usually include a statement of the form: "the estimated uncertainties quoted apply to the above tabulated values and should not be construed as being indicative of the long-term stability of the device under test." This 
statement is also important for the compressed gas insulated capacitors whose values might change significantly by handling during shipping.

The actual uncertainty quoted to the client is derived by algebraically summing the systematic uncertainties and adding three times the root mean sum of squares of the random uncertainties. For the capacitance measurement of compressed gas insulated capacitors, the measurement uncertainty will include a $20 \mathrm{ppm}$ contribution because of the possible $1 \mathrm{~K}$ variation in temperature of the NIST voltage transformer laboratory. For power-factor capacitors the self-heating variations will dominate ambient temperature effects.

\section{Appendix}

The voltage transformer will be represented as an ideal transformer with some unknown series output impedance $Z_{0}$, as shown in figure 9. The model has been shown to be sufficiently accurate experimentally. The relationship between the input voltage $E_{\mathrm{i}}$ and the output voltage with zero burden $E_{0}$ is:

$$
\frac{E_{\mathrm{i}}}{E_{0}}=N R C F_{0} e^{-j \Gamma_{0}},
$$

where $N$ is the nominal (or turns) ratio of the transformer, $R C F_{0}$ is the ratio-correction factor $\left(N \times R C F_{0}=\right.$ actual ratio) at zero burden, $\Gamma_{0}$ is the angle by which the secondary voltage vector leads the primary voltage vector, and $j=\sqrt{-1}$. A similar relationship exists between the input voltage $E_{\mathrm{i}}$ and the output voltage $E_{\mathrm{c}}$, with secondary burden $C$ (having impedance $Z_{\mathrm{c}}$ ) shown in figure 10 :

$$
\frac{E_{\mathrm{i}}}{E_{\mathrm{c}}}=N R C F_{\mathrm{c}} e^{-j \Gamma_{\mathrm{c}}}
$$

where $R C F_{\mathrm{c}}$ is the ratio correction factor with secondary burden $C$ and $\Gamma_{\mathrm{c}}$ is the corresponding phase angle.

Equating the current through $Z_{0}$ and $Z_{\mathrm{c}}$ in figure 10 , one obtains

$$
\frac{E_{0}-E_{\mathrm{c}}}{Z_{0}}=\frac{E_{\mathrm{c}}}{Z_{\mathrm{c}}}
$$

or

$$
E_{\mathrm{c}}=\frac{E_{0} Z_{\mathrm{c}}}{Z_{0}+Z_{\mathrm{c}}} .
$$

This can be rewritten in the following form:

$$
\frac{E_{\mathrm{i}}}{E_{\mathrm{c}}}=\frac{E_{\mathrm{i}}}{E_{0}}\left(1+\frac{Z_{0}}{Z_{\mathrm{c}}}\right)
$$

Setting $Z_{0}$ equal to $R_{0}+j X_{0}$ and $Z_{\mathrm{c}}$ equal to $R_{\mathrm{c}}+j X_{\mathrm{c}}$, eq (25) becomes:

$$
\frac{E_{\mathrm{i}}}{E_{\mathrm{c}}}=\frac{E_{\mathrm{i}}}{E_{0}}\left[1+\frac{R_{0}+j X_{0}}{R_{\mathrm{c}}+j X_{\mathrm{c}}}\right] .
$$

Taking the absolute value of both sides of eq (26), one finds that:

$$
\left|\frac{E_{\mathrm{i}}}{E_{\mathrm{c}}}\right| \approx\left|\frac{E_{\mathrm{i}}}{E_{0}}\right|\left[1+\frac{R_{0} R_{\mathrm{c}}+X_{0} X_{\mathrm{c}}}{R_{\mathrm{c}}^{2}+X_{\mathrm{c}}^{2}}\right],
$$

where it has been assumed that both $R_{0}$ and $X_{0}$ are much less than $Z_{\mathrm{c}}$ so that terms of order $\left[\left(R_{0} R_{\mathrm{c}}+X_{0} X_{\mathrm{c}}\right) /\left(R_{\mathrm{c}}^{2}+X_{\mathrm{c}}^{2}\right)\right]^{2}$ and higher have been neglected. Using eqs (21) and (26), one obtains

$$
\frac{E_{\mathrm{i}}}{E_{\mathrm{c}}}=\left|\frac{E_{\mathrm{i}}}{E_{0}}\right| e^{-j \Gamma_{0}}\left[1+\frac{R_{0}+j X_{0}}{R_{\mathrm{c}}+j X_{\mathrm{c}}}\right]
$$

or

$$
\frac{E_{\mathrm{i}}}{E_{\mathrm{c}}}=\left|\frac{E_{\mathrm{i}}}{E_{0}}\right| e^{-j \mathrm{r}_{0}}\left[1+\frac{\left(R_{0}+j X_{0}\right)\left(R_{\mathrm{c}}-j X_{\mathrm{c}}\right)}{R_{\mathrm{c}}^{2}+X_{\mathrm{c}}^{2}}\right] .
$$

This can also be expressed as

$$
\left|\frac{E_{\mathrm{i}}}{E_{\mathrm{c}}}\right| e^{-j \Gamma_{\mathrm{c}}}=\left|\frac{E_{\mathrm{i}}}{E_{0}}\right| e^{-j \Gamma_{0}}[\ldots] .
$$

Both exponentials have arguments much less than one so that discarding quadratic and higher order terms and equating the imaginary components of the left and right sides of eq (10) one obtains

$$
\left|\frac{E_{\mathrm{i}}}{E_{\mathrm{c}}}\right| \Gamma_{\mathrm{c}} \approx\left|\frac{E_{\mathrm{i}}}{E_{0}}\right|\left[\Gamma_{0}+\frac{X_{\mathrm{c}} R_{\mathrm{o}}-X_{0} R_{\mathrm{c}}}{R_{\mathrm{c}}^{2}+X_{\mathrm{c}}^{2}}\right]
$$

or from eq (5) assuming $Z_{0} \ll Z_{\mathrm{c}}$

$$
\Gamma_{\mathrm{c}} \approx \Gamma_{0}-\frac{X_{0} R_{\mathrm{c}}-X_{\mathrm{c}} R_{0}}{R_{\mathrm{c}}^{2}+X_{\mathrm{c}}^{2}}
$$

The resistive and reactive components of the burden $C$ can be expressed as

$$
R_{\mathrm{c}}=\sqrt{R_{\mathrm{c}}^{2}+X_{\mathrm{c}}^{2}} \cos \theta_{\mathrm{c}}
$$

and

$$
X_{\mathrm{c}}=\sqrt{R_{\mathrm{c}}^{2}+X_{\mathrm{c}}^{2}} \sin \theta_{\mathrm{c}},
$$

where $\cos \theta_{c}$ is the power factor of the burden $C$. From eqs (21) and (22)

and

$$
\left|\frac{E_{\mathrm{i}}}{E_{0}}\right|=N R C F_{0}
$$

$$
\left|\frac{E_{\mathrm{i}}}{E_{\mathrm{c}}}\right|=N R C F_{\mathrm{c}} .
$$


Using eqs (27) and (33)-(36) one obtains

$$
R C F_{\mathrm{c}}=R C F_{0}\left[1+\frac{1}{\left|Z_{\mathrm{c}}\right|}\left(R_{0} \cos \theta_{\mathrm{c}}+X_{0} \sin \theta_{\mathrm{c}}\right)\right]
$$

For the purposes of this discussion, it will be assumed that burden $C$ (having impedance $Z_{\mathrm{c}}$ ) above is the burden for which the ratio correction factor and phase angle are to be calculated. The ratio-correction factor and phase angle must be known for some other burden $T$, which shall be designated as having impedance $Z_{t}$. Using eq (25) and substituting burden $T$ for burden $C$ :

$$
Z_{0}=\left[\frac{E_{\mathrm{i}} / E_{\mathrm{t}}}{E_{\mathrm{i}} / E_{0}}-1\right] Z_{\mathrm{t}}
$$

or using eq (22)

$$
Z_{0}=Z_{\mathrm{t}}\left[R C F_{\mathrm{t}} e^{-j\left[\Gamma_{\mathrm{t}}-\Gamma_{0}\right]}-R C F_{0}\right] / R C F_{0} .
$$

Neglecting second order and higher terms

$$
Z_{0} \approx Z_{\mathrm{t}}\left[R C F_{\mathrm{t}}-R C F_{0}+j\left(\Gamma_{0}-\Gamma_{\mathrm{t}}\right)\right] / R C F_{0} .
$$

Using the facts that

$$
Z_{\mathrm{t}}=\left|Z_{\mathrm{t}}\right|\left(\cos \theta_{\mathrm{t}}+j \sin \theta_{\mathrm{t}}\right)
$$

and

$$
Z_{0}=R_{0}+j X_{0}
$$

one finds

$$
\begin{aligned}
R_{0} \approx\left(\frac{\left|Z_{t}\right|}{R C F_{0}}\right)\left[\left(R C F_{\mathrm{t}}-R C F_{0}\right) \cos \theta_{\mathrm{t}}\right. \\
\left.\quad+\left(\Gamma_{\mathrm{t}}-\Gamma_{0}\right) \sin \theta_{\mathrm{t}}\right]
\end{aligned}
$$

and

$$
\begin{gathered}
X_{0} \approx\left(\frac{\left|Z_{\mathrm{t}}\right|}{R C F_{0}}\right)\left[\left(\Gamma_{0}-\Gamma_{\mathrm{v}}\right) \cos \theta_{\mathrm{t}}\right. \\
\left.+\left(R C F_{\mathrm{t}}-R C F_{0}\right) \sin \theta_{\mathrm{t}}\right] .
\end{gathered}
$$

Using eqs (37), (43), and (44) and the relations:

$$
\begin{aligned}
& \cos \theta_{c} \cos \theta_{\mathrm{t}}+\sin \theta_{\mathrm{c}} \sin \theta_{\mathrm{t}}=\cos \left(\theta_{\mathrm{t}}-\theta_{\mathrm{c}}\right) \\
& \cos \theta_{\mathrm{c}} \sin \theta_{\mathrm{t}}-\sin \theta_{\mathrm{c}} \cos \theta_{\mathrm{t}}=\sin \left(\theta_{\mathrm{t}}-\theta_{\mathrm{c}}\right)
\end{aligned}
$$

one finds

or

$$
\begin{aligned}
R C F_{\mathrm{c}} \approx & R C F_{0}+\left(\frac{B_{\mathrm{c}}}{B_{\mathrm{t}} R C F_{0}}\right)\left[\left(R C F_{\mathrm{t}}-R C F_{0}\right)\right. \\
& \left.\cos \left(\theta_{\mathrm{t}}-\theta_{\mathrm{c}}\right)+\left(\Gamma_{\mathrm{t}}-\Gamma_{0}\right) \sin \left(\theta_{\mathrm{t}}-\theta_{\mathrm{c}}\right)\right]
\end{aligned}
$$

$$
\begin{array}{r}
R C F_{\mathrm{c}} \approx R C F_{0}+\left(\frac{B_{\mathrm{c}}}{B_{\mathrm{t}}}\right)\left[\left(R C F_{\mathrm{t}}-R C F_{0}\right)\right. \\
\left.\cos \left(\theta_{\mathrm{t}}-\theta_{\mathrm{c}}\right)+\left(\Gamma_{\mathrm{t}}-\Gamma_{0}\right) \sin \left(\theta_{\mathrm{t}}-\theta_{\mathrm{c}}\right)\right]
\end{array}
$$

where $B_{\mathrm{c}}=1 / Z_{\mathrm{c}}$ is the burden in $\Omega^{-1}$ of the impedance $Z_{\mathrm{c}}$. Since the second term in eq (47) represents a small correction to the first and since $R C F_{0}$ is approximately equal to one, $R C F_{0}$ has been dropped from the second term of eq (48). Using eqs (32)-(34)

$$
\Gamma_{\mathrm{c}} \approx \Gamma_{0}-\frac{1}{\left|Z_{\mathrm{c}}\right|}\left(X_{0} \cos \theta_{\mathrm{c}}-R_{0} \sin \theta_{\mathrm{c}}\right) .
$$

Using eqs (43)-(46) and (49)

$$
\begin{aligned}
\Gamma_{\mathrm{c}} \approx & \Gamma_{0}+\left(\frac{B_{\mathrm{c}}}{B_{\mathrm{t}} R C F_{0}}\right)\left[\left(\Gamma_{\mathrm{t}}-\Gamma_{0}\right) \cos \left(\theta_{\mathrm{t}}-\theta_{\mathrm{c}}\right)\right. \\
& \left.-\left(R C F_{\mathrm{t}}-R C F_{0}\right) \sin \left(\theta_{\mathrm{t}}-\theta_{\mathrm{c}}\right)\right]
\end{aligned}
$$

or

$$
\begin{aligned}
\Gamma_{\mathrm{c}} \approx & \Gamma_{0}+\left(\frac{B_{\mathrm{c}}}{B_{\mathrm{t}}}\right)\left[\left(\Gamma_{\mathrm{t}}-\Gamma_{0}\right) \cos \left(\theta_{\mathrm{t}}-\theta_{\mathrm{c}}\right)\right. \\
& \left.-\left(R C F_{\mathrm{t}}-R C F_{0}\right) \sin \left(\theta_{\mathrm{t}}-\theta_{\mathrm{c}}\right)\right]
\end{aligned}
$$

since $R C F_{0}$ is approximately equal to one.

Equations (48) and (51) can be used to calculate the $R C F$ and phase angle for some secondary bur$\operatorname{den} C$, if the ratio correction factors and phase angles are known at some other burden $T$, and at zero burden.

\section{Acknowledgments}

The author would like to thank Oskars Petersons, Chief of the Electrosystems Division, who has been the source of nearly all the author's knowledge on the calibration of voltage transformers and capacitors. The author would also like to acknowledge the work of Barbara Frey and Roberta Cummings who helped prepare this manuscript. Last, but certainly not least, the author would like to acknowledge both the "old-timers" here at the National Institute of Standards and Technology, who began the tradition of excellence in measurements, and the present calibration staff who are attempting to carry on this tradition under vastly different constraints.

About the author: William E. Anderson is a physicist in the Electrosystems Division of the NIST Center for Electronics and Electrical Engineering. 


\section{References}

[1] Harris, F. K., Electrical Measurements, John Wiley \& Sons, New York (1966) pp. 576-577.

[2] Harris, F. K., Electrical Measurements, John Wiley \& Sons, New York (1966) pp. 687-738.

[3] Kusters, N. L., and Petersons, O., Trans. Commun. Electron. (U.S.) CE-82 606 (1963).

[4] McGregor, M. C., Hersh, J. F., Cutkosky, R. D., Harris, F. K., and Kotter, F. R., Trans. on Instrum. (U.S.) I-7 No. 3 and 4 (1958).

[5] Hillhouse, D. L., and Peterson, A. E., IEEE Trans. Instrum. Meas. (U.S.) IM-22 406 (1973).

[6] Anderson, W. E., Davis, R. S., Petersons, O., and Moore, W. J. M., IEEE Trans. Power Appar. Syst. (U.S.) PAS-97 1217 (1973).

[7] IEEE Standard Requirements for Instrument Transformers, American National Standards Institute, ANSI/IEEE C57.13-1978 32 (1978).

[8] IEEE Standard Requirements for Instrument Transformers, American National Standards Institute, ANSI/IEEE C57.13-1978 45 (1978).

[9] Harris, F. K., Electrical Measurements, John Wiley \& Sons, New York (1966) pp. 673-687.

[10] Petersons, O., and Anderson, W. E., IEEE Trans. Instrum. Meas. (U.S.) IM-24 4 (1975).

[11] Petersons, O., A Wide-Range High-Voltage Capacitance Bridge with One PPM Accuracy, D.Sc. dissertation, School of Engineering and Applied Sciences, George Washington University, Washington, DC (1974). 